stimulus, and is linked up to it through a long chain. The alarm clock rings; we do not get up, and it ceases to stimulate us, to call out the conditioned reflex of arising. The unconditioned stimulus here is not so easy to detect as in the dog which fails to react to the bell connected with food, and it is probably a compound one (the fear of falling into disrepute, losing of one's job, meeting with disapproval-which is quite likely an unconditioned stimulus for man just as an overpowering frightful enemy is for an animal).

Again :

" Seize the very first opportunity to act on every resolution you make, and on every emotional prompting you may experience, in the direction of the habits you aspire to gain. It is not in the moment of their forming, but in the moment of their producing motor effects [that is, conditioned reflexes]. when resolves and aspirations communicate the new set to the brain. . . . No matter how full a reservoir of maxims one may possess, and no matter how good one's sentiments may be, if one has not taken advantage of every concrete opportunity to act, one's character may remain entirely unaffected for the better."

This is just the same way of stating the preceding rule from the standpoint of conditioned reflex elaboration. James's final rule of " keeping the faculty of effort alive in you by a little gratuitous exercise every day" involves both the emotional state and the value of repetition in the elaboration of conditioned reflexes and in the prevention of their gradual spontaneous extinction.

\section{Morals and Prejudices}

Besides these maxims we wish to make several direct applications from the study of conditioned reflexes to habit formation. It will be seen that after the first conditioned reflex in a certain centre is elaborated it takes fewer trials for each succeeding one up to a certain point. This is a basic law of education. That extinction of the second conditioned reflex is much easier than of the first, and that when once it has been extinguished and restored it is never so stable as it was before extinction, has an enormous significance in the destruction of moral codes, explaining why a person untrustworthy in one thing is usually so in another, the chaos produced by revolutions and wars, the difficulty of bringing about permanent cures in drunkards, culprits, etc.

How strong the old conditioned reflexes are and their spontaneous restoration after extinction, and how quickly recently elaborated conditioned reflexes disappear, is seen in the readiness with which habits cultivated late in life are abandoned, and in how inevitably and easily a man who has acquired new manners and culture removed from his early surroundings lapses back into his former habits, ungrammatical slips of the tongue, accent, etc. (though they may not have been used for years), when he returns to the old situation. The fact that a delayed reflex is weaker than an immediate one explains the demoralizing effect of the action of procrastination, and the nervous tension (conflict between excitation and inhibition) when rewards (unconditioned stimuli) are too long withheld in those unaccustomed to maintaining a balance between the processes of excitation and inhibition. And cannot many of our prejudices find an explanation in the phenomena of conditioned inhibition? Here all accidental stimuli or changes in the environment which may be entirely without significance for the organism will, if they coincide with an inhibitory state in the animal, after a certain number of coincidences of times provoke the same state when they occur alone.

\section{Danger of Repeated Stimul}

Perhaps we might learn something from the laboratory concerning the repetition of conditioned reflexes. Too frequent use of the same conditioned stimulus may become injurious. Instead of leading to the successful elaboration and establishment of the desired conditioned reflex (habit), its weakening and disappearance may ensue - that is, it may pass over into an inhibitory agent despite reinforcement each time. This is especially true of certain kinds of weak stimuli (tactile and thermal for the dog). Between such failing stimuli it is necessary to interpose other positive conditioned stimuli, or, better, negative conditioned stimuli, which evoke the process of induction causing the opposite stimuli to act better. These interposed conditioned stimuli may be ones connected with the same unconditioned reflex.

\section{Conditioned Reflexes and Behaviour}

The behaviour of man can be viewed from the standpoint of Pavlov as a long chain of conditioned reflexes. In the laboratory we can form chains of only two or three conditioned reflexes in the dog-that is, where one signal is not the immediate signal for the unconditioned stimulus but for another conditioned stimulus, and so on through the chain of conditioned stimuli till the unconditioned stimulus is reached. In the child, chains of much greater length have been elaborated. The child is more plastic than the dog, and in it conditioned reflexes are more readily and more easily lost. This is true for the food reflex, but there is doubtless a variation for the different unconditioned reflexes. The fund of unconditioned reflexes varies markedly in each animal, so that the results obtained by working with one unconditioned reflex may not be comparable in different species as a measure of intelligence, capability, etc. A child would hardly be expected to establish easily a positive conditioned food reflex to a worm, nor a fish as readily as a child an investigating reflex to a drawing on a piece of white paper.

Although Pavlov has opened up a vast untrodden territory for exploration by his method of conditioned reflexes, and though we may find it of great aid in the investigation of psycho-biologic fields, we must remember that niuch caution and perseverance and further study is necessary before we can use it with confidence in the general explanation of psycho-biologic reactions (behaviour). He says: "It would be the height of presumption to regard these first steps in elucidating the physiology of the cortex as solving the intricate problem of the higher psychic activity in man, when in fact at the present stage of our work no detailed application of its results to man is yet permissible."

\section{THORACIC METASTASES IN OVARIAN CARCINOMA}

Report of a Case with Remarks

BY

HERBERT T. EVANS, M.D., M.R.C.P. ASSISTANT PHYSICIAN, CARDIFF ROYAL INFIRMARY AND

GILBERT I. STRACHAN, M.D., F.R.C.P., F.R.C.S., F.C.O.G.

GYNAECOLOGIST, CARDIFF ROYAL INFIRMARY

The particular feature of the case to be described is that all the symptoms and signs were pulmonary, and for this reason the patient was admitted to, and treated in, a medical ward by one of us $(\mathrm{H}$. T. E.). There were no abdomino-pelvic signs of any physical abnormality, and only on post-mortem examination was the primary ovarian lesion made evident.

\section{Clinical History}

The patient, aged 53 years, was seen on July 6th, 1931, in the out-patient department, and was admitted to hospital the same day. 
The history given was one of increasing malaise since the beginning of the year. Palpitation and dyspnoea on exertion gradually appeared, while for the last two months there had been pain over the right side of the chest. This had been diagnosed as pleurisy. The pain was described as beginning in the back, gradually working round to the lower ribs of the right side and the right costal margin, and extending down the right flank, while the dyspnoea was becoming more marked. There was very little cough, and practically no sputum. The appetite was poor, the bowels regular; no vomiting, and no urinary trouble. Sleep was often disturbed by the pain. She usually felt worse about 5 a.m. and 5 p.m. She thought she was losing weight. There was occasional swelling of the ankles towards evening.

The patient was married at an early age, and had never had any children. The menopause appeared when she was 42 years old. She had an operation for "womb trouble" thirty years ago, and was subject to bronchitis nearly every winter.

Examination showed a stout woman, with a somewhat cyanosed face, and dyspnoeic; respirations 24 per minutc. She looked ill, and perspired freely. The voice was husky, and she spoke as if with difficulty. The most comfortable position was resting half propped up in bed.

The heart was normal in size and position, with no murmurs; first sound at apex rather accentuated; rhythm regular, rate 104 per minute.

Inspection of the chest showed no asymmetry or abnormality other than delay and limitation of movement of the right side, more so over the lower part, and this was confirmed by palpation. On percussion there was marked dullness to the top of the fourth rib in front, and posteriorly to just above the root of the lung. The breath sounds were much diminished over the more anterior portion of the above area, nearly absent over the axillary and posterior portions. Above the level of dullness only slight signs of compression were present. There was no friction rub. The left lung showed the ordinary signs of compensation. The lower part of the right chest, particularly in front, was very tender, and so also was the costal margin.

The abdomen moved freely, and was not distended. There was no abnormal dullness, no rigidity, no palpable tumour, and no tenderness, except along the right costal margin. The liver was not enlarged, and the spleen could not be palpated. The urine contained no albumin, and no oedema was to be seen.

On the day following admission to hospital the right chest was aspirated, and several ounces of blood-stained fluid were removed. Microscopical examination showed " clumps of cells " very suggestive of malignancy. The fluid contained also an excess of albumin, while culture showed only a few staphylococci.

Considerable relief was experienced after the aspiration, the dyspnoea and cyanosis diminishing, while the physical signs over the upper lobe became more normal. This condition continued for the next five days, when the temperature rose to $101.8^{\circ} \mathrm{F}$. and the dyspnoea increased but the cyanosis remained unchanged. At the end of twenty-four hours the temperature dropped. The pain along the costal margin was now alnost continuous, though less severe. Soreness was experienced in the back, round the site of aspiration, and a small, rather firm swelling with a reddened centre began to develop. This slowly increased in size, retained its firmness, and appeared fixed. The surrounding tissues became indurated, and the whole mass was very tender and painful. There was no pitting on pressure. The temperature continued about normal, and the pulse showed no variation. With the development of the tumour a solitary enlarged gland was found in the right supraclavicular fossa. Two attempts were made to $x$-ray the chest, but on each occasion the patient collapsed on arriving at the department, so that no information from this point of view was obtained.

The cyanosis became more marked. A second aspiration was performed a month after the first, as the fluid had rapidly increased and was causing much dyspnoea. This brought temporary relief, and during the interval the breath sounds could be heard over the upper lobe in front and as far down as the seventh rib behind, but the chest movements did not improve. The pain along the costal margin had now become continuous. The swelling in the back was still pain- ful, but unchanged in size ; its outline remained indefinite. No further glandular enlargements could be found, and the liver showed no increase in size. The patient gradually became weaker, but did not seem to be losing weight rapidly. The end came very suddenly; the patient, who had been talking cheerfully, all at once collapsed, and died within a couple of minutes.

The diagnosis suggested initially was neoplasm of the lung with pleural effusion. The question of malignancy received confirmation from the report on the pleural fluid. A point of great interest was the advent of the tumour over the site of puncture for aspiration. This was adjudged to be also malignant in view of the absence. of pyrexia, disturbance of pulse rate, and profuse sweats. For this reason the tumour was not disturbed.

\section{Post-Mortem Findings}

At necropsy the most evident lesions were thoracic. In the right chest carcinomatous deposits were present on the diaphragmatic pleura and on the parietal surface, but to a lesser degree, and mainly in relation to the inner aspects of the ribs. The visceral pleura was thickened, but showed no evident malignant deposits. There was a large quantity of blood-stained fluid in the pleural cavity, so that the lung was collapsed and completely shrunken.

On the left side the pleura was normal, and the lung, apart from some emphysema towards the anterior edges, was also apparently normal. The anterior mediastinum was extensively infiltrated with growth, obviously malignant. A certain amount of free turbid fluid was present in the abdomen, while the mesentery and omentum were completely infiltrated with growth similar to that found in the chest, but more extensive.

The alimentary tract, liver, gall-bladder, and kidneys were free, but some injection of the gastric mucosa was present.

The uterus and cervix were found normal, but the right ovary was enlarged to about the size of a tangerine orange, and of irregular shape. It appeared on section to be composed mainly of oedematous fibrous tissue, with breaking-down patches, and also mucoid areas, scattered throughout it. The left ovary was enlarged, but not so much as the right; its contents on section were of a similar fibro-mucoid character.

On microscopical examination the ovarian tissue was largely occupied by masses of carcinoma cells which, in some areas, retained an original adenocarcinomatous appearance, but, owing to proliferative growth and anaplastic retrogression of the cells, this appearance had been destroyed over the greater part of the sections, and merely accumulations of masses of carcinoma cells of various shapes and sizes were seen, with frequent mitotic figures.

In sections from the secondary growths a similar extreme degree of infiltration with carcinomatous cells was seen. They were of identical appearance with those of the ovary, and with the tendency to be arranged in definite groups rather than to be generally infiltrative throughout the tissue.

This case shows the importance of seeking carefully for a primary focus in cases of thoracic carcinoma. But a more important feature here was the lack of any physical signs of abdominal involvement, although later examination showed free fluid and extensive omental and mesenteric involvement.

The insidious growth and extension characteristic of so many ovarian malignant tumours is well illustrated by this case, and it is in the experience of all gynaecologists that patients well nourished and complaining of but few symptoms present themselves from time to time in whom evidence of widespread peritoneal involvement is found.

A point worthy of consideration is whether the growth was primarily ovarian, or whether the ovaries were 
involved secondarily by transcoelomic transplantation of carcinoma cells from some unsuspected primary focus. The main primary foci in this connexion are the stomach and intestines, and these structures were here found to be normal, as well as the breast, from which a lymphatic and later transcoelomic spréad may occur. Again, the microscopical features were essentially those seen in ovarian carcinoma.

Cases of ovarian carcinoma with multiple metastases, in some of which the ovarian origin was unsuspected, have been reported from time to time. Thus Bankart ${ }^{1}$ in 1926 reported such a case in which the secondaries were in the scapula, while Simard and Gagnon ${ }^{2}$ in 1928 published a case in which secondary ovarian malignant tissue was found in the arm. In both of these cases microscopical examination of the secondary growth showed malignant tissue morphologically similar in structure to that found in the primary ovarian growth. In Muller's case, ${ }^{3}$ reported in 1929, multiple extensions were present in the serous membranes, with a microscopical structure resembling mesothelioma, while Cavour ${ }^{4}$ in 1920 and van Dongen ${ }^{5}$ in 1922 described similar cases in which the ovarian origin was unsuspected.

We are indebted to Professor Duguid for the post-mortem and histological material concerned in the case.

\section{ReFERENCES}

'Bankart, Blundell: Proc. Roy. Soc. Med., Sect. Orth., 1926, xix, 12

Simard, L. G., and Gagnon, A.: Union Méd. du Canada, 1928, lvii, 145 .

Muller, G.: Tumori, 1929, xv, 170

- Cavour, T.: Gynécol. et Obstét., 1920, i, 71.

san Dongen, J. A.: Nederl. Tijdschr. v. Geneesk., 1922, i, 1630.

\section{Memoranda}

\section{MEDICAL, SURGICAL, OBSTETRICAL}

\section{ELEVATION OF DEPRESSED FRACTURE OF THE SKULL IN A NEWBORN CHILD}

The following case may be of some interest. Mrs. MacX. is a case of flat pelvis. She has had six children-four stillborn, one died after fourteen days, and one is still alive as the result of induction of premature labour. Most of those children, from what I can gather, died of fractured skull. Certainly the child who lived a fortnight had a severe skull injury, and suffered from fits during its short life. One which I delivered had a definite depressed fracture of the skull, but was stillborn.

On the morning of January 28th, 1932, I learned that Mrs. MacX. was again pregnant and almost due. I went to see her at once, and on bimanual examination found that disproportion was marked. With quinine sulphate, castor oil, and pituitrin I induced premature labour, and delivered an asphyxiated female child, weighing eight pounds, at 5.40 a.m. on the following day.

It was a high forceps case, and the head seemed as if it would not enter the pelvis. Then something cracked, and the head came through easily and was delivered. A large depressed fracture of the left frontal bone $2 \frac{1}{2}$ inches long by 2 inches at its widest margins became evident. It had been caused by the promontory of the sacrum, and was angled upwards and backwards across the frontal bone. After some delay with the shoulders the child was ultimately born-stillborn as I thought; but the heart was beating, and after resuscitation it came round. There it was: the depression of the left frontal bone, a staring left eye, a flaccid right arm and leg in a semi-conscious infant.

Deciding upon immediate operation I boiled a small scalpel and a slim dissecting forceps used for packing tooth sockets. The scalp was prepared with iodine. A small incision was made over the lower margin of the left frontal bone ; but I could not get the handle of the forceps under the bone, so I made an incision half an inch long over the frontal suture one inch in front of the large fontanelle, and managed to get the handle of the forceps between the membranes and the bone. Gently but firmly I insinuated the forceps until under the central furrow of the depression. Then, with a lifting, levering movement of the forceps handle, the depression became elevated to the normal. Since the child bore the operation well, I boiled a small needle, one strand of silkworm-gut, and a pair of scissors, and completed the operation by putting one stitch in each incision.

Before I left this one-roomed home at 7 a.m. the child was crying lustily, shutting both eyes equally, and using both arms and legs freely. The child has been carefully watched; it takes the breast, has not been fevered, and has altogether made an uneventful recovery.

Huntly, Aberdeenshire. Peter W. Philip, M.B., Ch.B.

\section{SPONTANEOUS HAEMORRHAGE OF THE NEWBORN}

As the spontaneous haemorrhages of the newborn constitute such a grave emergency, and probably occur at some time in the experience of most family doctors, perhaps it will not be out of place for even a fourth-rate practitioner in a third-rate practice to record a case, with recovery.

\section{Report of a CASE}

The confinement, a first one, was normal and unassisted; I merely supervised progress. The whole course of the pregnancy was perfectly normal and uneventful. The family history and antecedents of the parents form a dull record of rude health and blameless conduct. The child was a boy, and seemed beyond criticism, until, a few hours after birth, he vamited a little faintly brown fluid, suggestive of a trace of altered blood. These two abnormalities-the vomiting and the colour-were repeated on the second day, so I gave a weak mixture of bismuth and salol. On the third day there was no vomiting, and the stools seemed to me to be quite normal. But I was not altogether surprised, when called in hurriedly while visiting nearby on the fourth day, to find the child blanched, pulseless, and quite collapsed-apparently dead, until I felt a faint apex beat-with his gown saturated in front with bright-red blood from the mouth, and the napkin full of tarry stools.

With a syringe I drew blood from the mother and injected the greater part of $10 \mathrm{c} . \mathrm{cm}$. into the deeper tissue of the child's buttocks and thighs. The baby began to revive, and I asked that he should be given sweetened water freely, his mixture as before, and that he should be kept as warm and undisturbed as possible. Later, I added a few drops of liq. adrenalin. hydrochlor. to the mixture, but the child had recovered sufficiently to recognize and resent this addition. Next morning there was a further haemorrhage, so I gave another injection of the mother's blood. I used a very widebore intramuscular needle, and had the barrel of the syringe filmed over inside with soft paraffin, as I found that otherwise it was likely to " seize." In the evening I gave a third injection, and also injected a rough fourth of a $5 \mathrm{c.cm}$. ampoule of a new liver extract I happened to have in my bag. Next day there was no haemorrhage, but I gave a similar injection of the liver extract, and repeated it on the two succeeding days. Thereafter the child was doing so well that treatment seemed superfluous-he was suckling well, sleeping well, and having normal bowel action. A day or two later he was out of doors, and in a couple of weeks seemed no more anaemic than many babies of that age.

Another time, confronted with a brownish tint in fluid vomited within a few hours of birth, I should not hesitate to inject whole blood, or even any of the usual serums, if parents' blood could not be got. Waiting is too dangerous, and only good luck saved this child. Nothing could be simpler or cheaper. The liver extract may have 